\title{
Third Ventricular Glioblastoma Multiforme: Case Report and Literature Review
}

\author{
Omid R. Hariri ${ }^{1,2}$ Syed A. Quadri ${ }^{1,2}$ Saman Farr ${ }^{3}$ Ravi Gupta ${ }^{4}$ Andrew J. Bieber ${ }^{1,2}$ \\ Anya Dyurgerova ${ }^{3}$ Casey Corsino $^{3}$ Dan Miulli $^{1}$ Javed Siddiqi, ${ }^{1,2}$
}

\footnotetext{
${ }^{1}$ Department of Neurosurgery, Arrowhead Regional Medical Center, Colton, California, United States

${ }^{2}$ Department of Neurosurgery, Institute of Clinical Orthopedics and Neurosciences, Desert Regional Medical Center, Palm Springs, California, United States

${ }^{3}$ Division of Neurosurgery, Department of Surgery, College of Osteopathic Medicine, Western University of Health Sciences, Pomona, California, United States

${ }^{4}$ Department of Radiology, Harbor-UCLA Medical Center, Torrance, California, United States
}

Address for correspondence Omid R. Hariri, DO, MSc, Department of Neurosurgery, Arrowhead Regional Medical Center, Colton, CA 92324, United States (e-mail: ohaririucla@gmail.com).

J Neurol Surg Rep 2015;76:e227-e232.

\begin{abstract}
Keywords

- glioblastoma multiforme

- third ventricle

- foramen of Monroe

- gliosarcoma

- septum pellucidum

- hydrocephalus

- fornix

Background Glioblastoma multiforme (GBM) typically presents in the supratentorial white matter, commonly within the centrum semiovale as a ring-enhancing lesion with areas of necrosis. An atypical presentation of this lesion, both anatomically as well as radiographically, is significant and must be part of the differential for a neoplasm in this anatomical location.

Case Description We present a case of a 62-year-old woman with headaches, increasing somnolence, and cognitive decline for several weeks. Magnetic resonance imaging demonstrated mild left ventricular dilatation with a well-marginated, homogeneous, and nonhemorrhagic lesion located at the ceiling of the third ventricle within the junction of the septum pellucidum and fornix, without exhibiting the typical radiographic features of hemorrhage or necrosis. Final pathology reports confirmed the diagnosis of GBM.

Conclusion This case report describes an unusual location for the most common primary brain neoplasm. Moreover, this case identifies the origin of a GBM related to the paracentral ventricular structures infiltrating the body of the fornix and leaves of the septum pellucidum. To our knowledge this report is the first reported case of a GBM found in this anatomical location with an entirely atypical radiographic presentation.
\end{abstract}

\section{Background}

GBM is an intra-axial mass, localized to the frontotemporal region that accounts for 50 to $60 \%$ of astrocytomas and 12 to $15 \%$ of intracranial tumors. It is found most commonly as a primary tumor, with secondary tumor characteristics as result of transformation from low-grade glioma. ${ }^{1-8}$ Intraventricular tumors represent only $\sim 10 \%$ of central nervous system neoplasms. Of those, the reported malignancy rate is $\sim 13 \%$ of all cases. Lee and Manzano enumerated the most frequently found ventricular system tumors, presented in - Table 1. ${ }^{9}$ The rarest lesions of the intraventricular region are GBM, ependymomas, gangliocytomas, and gliosarcomas. ${ }^{10}$ Ependymomas occur at a frequency of $0.25 \%{ }^{11}$ and gangliocytomas with a frequency of 0.1 to $0.5 \% .^{12}$ When received

November 13, 2014

accepted

June 12, 2015

published online

August 4, 2015
DOI http://dx.doi.org/

10.1055/s-0035-1560048. ISSN 2193-6358. (c) 2015 Georg Thieme Verlag KG
Stuttgart · New York

License terms

((1) $\circledast$ 
Table 1 Tumors documented in the intracranial ventricular system ${ }^{7}$

\begin{tabular}{|l|l|}
\hline Neurocytoma & Pilocytic astrocytoma \\
\hline Meningioma & Oligodendroglioma \\
\hline Colloid cyst & Gangliocytoma \\
\hline Carcinoma & Malignant astrocytoma \\
\hline Ependymoma & Choroid plexus papilloma \\
\hline Germinoma & Metastasis \\
\hline Subependymoma & $\begin{array}{l}\text { Subependymal giant } \\
\text { cell astrocytoma }\end{array}$ \\
\hline Schwannoma & \\
\hline
\end{tabular}

found intraventricularly, GBM has a predilection for the frontal horn or body of the lateral ventricle. ${ }^{13}$ As shown in -Table 2, an extensive literature review resulted in only 13 identified cases of GBM (including the presently reported case) and 3 cases of gliosarcoma isolated to the third ventricle.

Histologically, the GBM cells appear pleomorphic and poorly differentiated with nuclear atypia and polymorphism, with a pseudo-cascading appearance. ${ }^{13,21}$ Hypercellularity, high mitotic activity, and vascular proliferation are further typical characteristics of GBM. Glial fibrillary acidic protein (GFAP) and Ki-67 antibodies are known nuclear antigen markers used to determine the proliferation fraction of gliomas. They are utilized in immunohistochemical staining and stain positive in GBMs. ${ }^{13,15}$

Radiographically, GBMs characteristically present as a heterogeneous mass with necrosis and ring enhancement. $^{12,22,23}$ The ring enhancement appearance is due to the presence of dense cellularity and neovascularization in the outer viable margin of the tumor, encompassing the T2-hyperintense necrotic region. ${ }^{23}$

To our knowledge, we are reporting the first case of a GBM localized to the roof of the third ventricle with atypical radiographic characteristics.

\section{Clinical Presentation}

\section{History}

A 62-year-old right-handed white woman presented with an altered level of consciousness accompanied by headaches, nausea, and vomiting for $\sim 4$ days, as reported by family members. This was preceded by worsening of short-term memory loss, increasing generalized confusion, increasing somnolence, decreased activity, and cognitive decline for several weeks. There were no reported visual changes, seizure activity, or gait disturbances reported. The patient had a past medical history of chronic obstructive pulmonary disease, diabetes mellitus, gastroesophageal reflux disease, hypertension, dyslipidemia, and congestive heart failure. She admitted to a history of tobacco use for 30 years but denied alcohol or recreational drug use.

\section{Examination}

Mental status: Patient opened eyes to voice; however, she appeared abulic. She laughed at times and also gestured appropriately at times. Cranial nerves: Pupils were $\sim 4 \mathrm{~mm}$ to $3 \mathrm{~mm}$, with no clear papilledema on the right, although without very distinct disk margins; OS with clear margins laterally only. Visual threat response was present bilaterally. Upper and lower facial movements were both symmetrical. Patient was not cooperative with subjective sensory questioning in the trigeminal distribution. Other cranial nerves were grossly intact. Motor: She provided a 5/5 grip, triceps, biceps, knee extension, ankle dorsiflexion, as well as hip and knee flexion. No fasciculations, tremors, or myoclonus was noted. Sensory: Light touch sensation was intact and symmetrical in the upper and lower extremities bilaterally, and proprioception was intact in the distal interphalangeal joints. Cerebellar: No nystagmus. No dysrhythmia of speech. Patient was not cooperative with appendicular targeted movements. Reflexes: Down-going responses with Babinski testing bilaterally.

\section{Radiographic Findings}

Computed tomography imaging of the head revealed round enlargement of the midline paraventricular structures without calcification, with associated mild hydrocephalus. The subsequent magnetic resonance imaging (MRI) with gadolinium showed thickening of the inferior septum pellucidum/ fornix (-Fig. 1C), with the width in the coronal plane measuring nearly $13 \mathrm{~mm}$ (normal width between 1.5 and $3.0 \mathrm{~mm}^{7}$ ). Two adjacent well-marginated T1-isointense and $\mathrm{T} 2$ mildly hyperintense masses were seen within the midline structures. Postgadolinium T1-weighted images showed corresponding mild homogeneous enhancement (-Fig. 1A, B).

There was a notable absence of restricted diffusion, gradientecho hyperintense signal (indicating lack of hemorrhagic products), central cystic/necrotic changes, or rim enhancement. There was left lateral ventricular moderate dilatation with periventricular fluid-attenuated inversion recovery hyperintense signal, reflecting transependymal flow suggestive for acute obstructive hydrocephalus. The third and fourth ventricles were of normal size, and the fast imaging employing steady-state acquisition sequence did not suggest an overt occlusion or increased flow in the cerebral aqueduct (-Fig. 1C).

\section{Operation}

A small right one-third frontal and two-thirds parietal standard transcallosal approach craniotomy with right transseptal approach toward the lesion was performed. The mass was located between the leaves of the septum pellucidum with bilateral forniceal infiltration and extension into the caudal aspect of the third ventricle. Grossly, the tumor appeared soft with a gray/purple color, without any evidence of venous thrombosis. The mass was microscopically identified intraoperatively, and a near-complete resection of the visible mass was achieved.

\section{Pathologic Findings}

Upon gross examination, the tumor appeared to have multiple pink-tan and gray soft tissue fragments. Tumor biopsy results revealed a cellular neoplasm. GFAP by immunohistochemistry was strongly positive. The Ki-67 by immunohistochemistry was high, with 50 to $80 \%$ nuclear staining. A 
Third Ventricular Glioblastoma Multiforme Hariri et al. e229

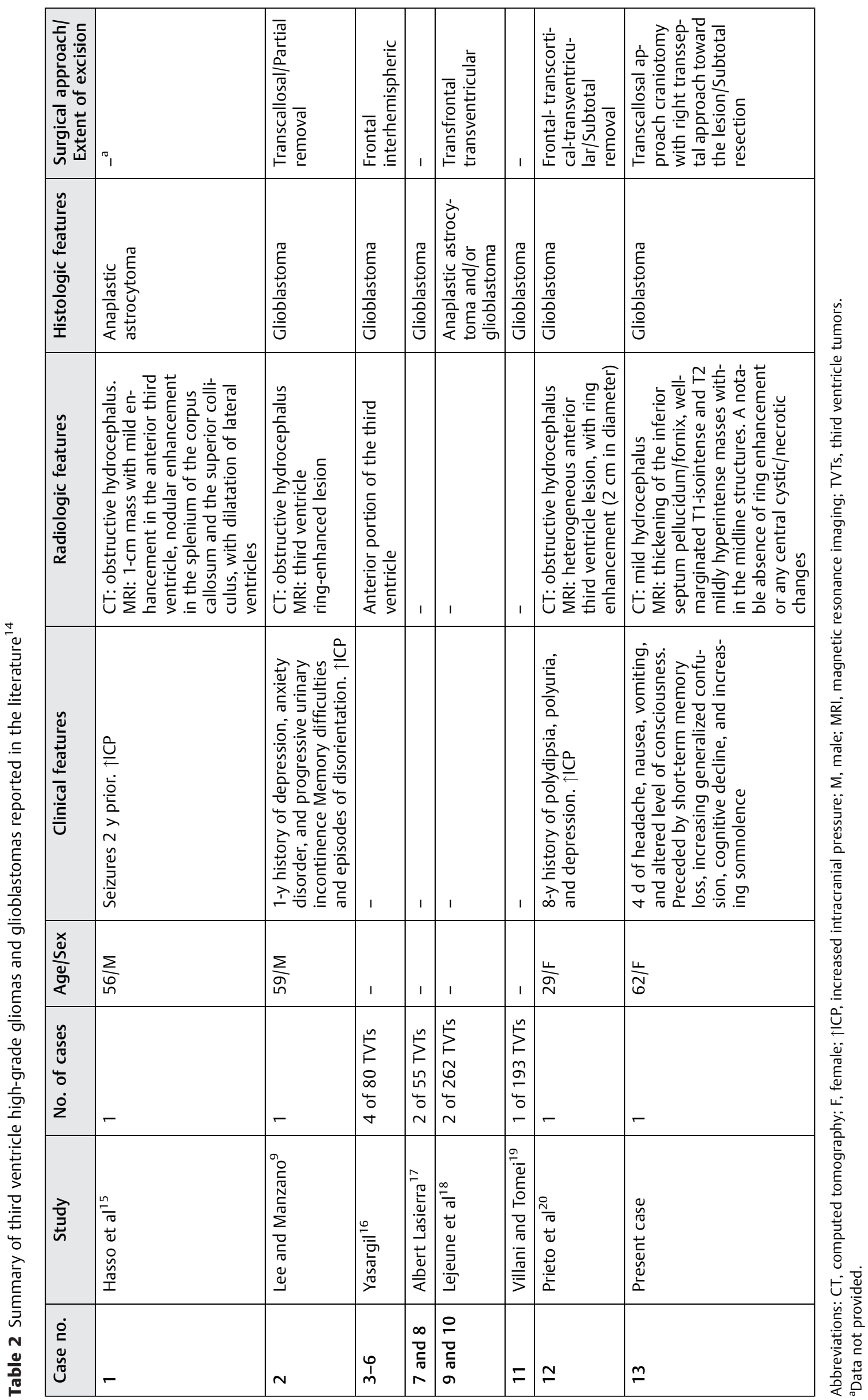



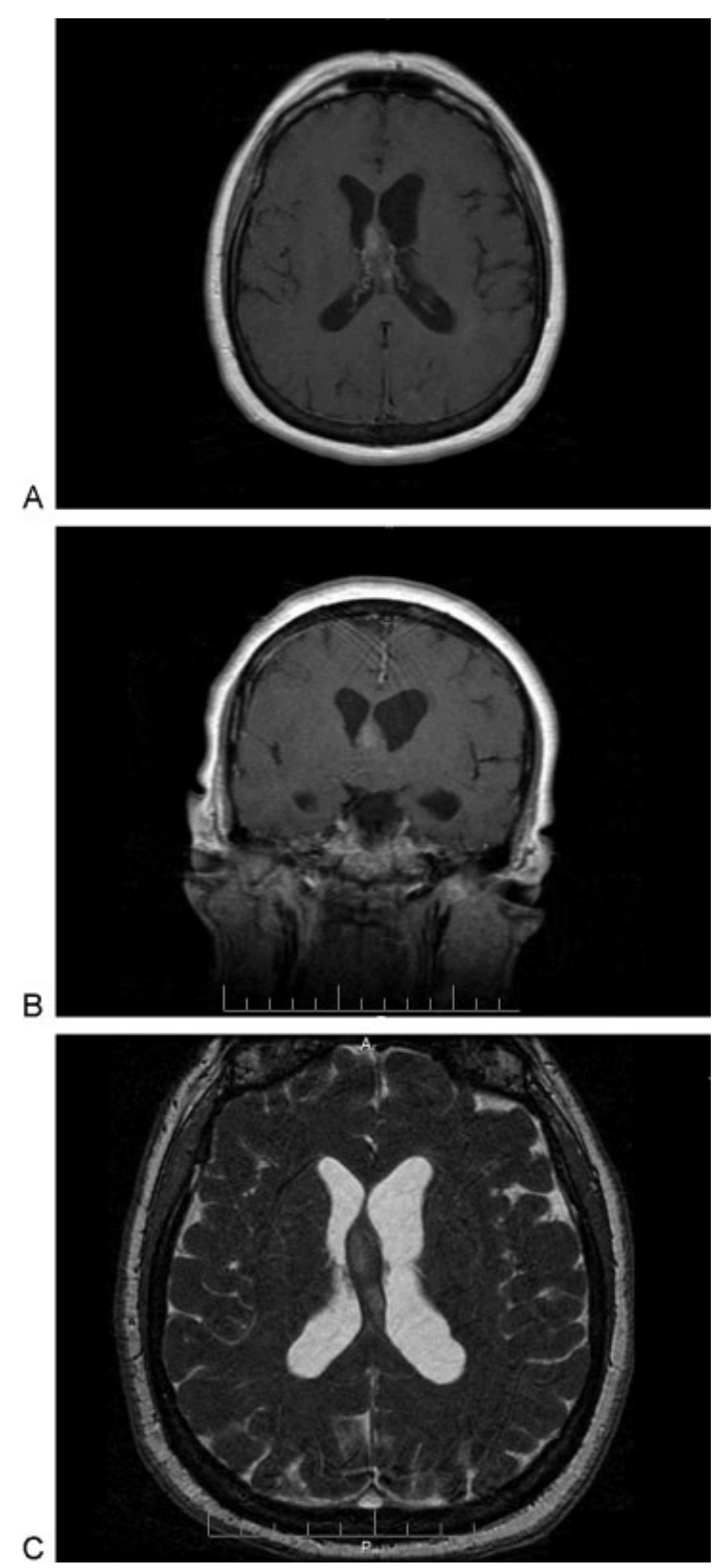

Fig. 1 (A) Preoperative contrast-enhancing T1-weighted magnetic resonance image (MRI). Axial image showing mild enhancement of the anterior lobular mass. (B) Preoperative contrast-enhancing T1-weighted MRI. Coronal image showing abnormal thickening of the inferior septum pellucidum and fornix to $1.3 \mathrm{~cm}$. (C) Preoperative T2-weighted fast imaging employing steady-state acquisition MRI. Axial image showing two mildly hyperintense adjacent lobular masses within and expanding the fornix.

serpentine pattern of necrosis and surrounding hypercellularity was present on histologic section, along with the characteristic pseudo-palisading pattern that occurs with GBM (-Fig. 2).

\section{Postoperative Course}

Postoperatively, the patient's mental status and generalized confusion improved. She appeared to be significantly less lethargic and able to participate in limited physical therapy activities. The patient's headaches, nausea, and vomiting also improved. The family noted a marked increase in cognition affect and ability postoperatively. Options of postoperative whole brain radiation and chemotherapy were discussed with the patient and her family. They decided that they did not want to continue with any aggressive or palliative treatment and chose comfort care only. The patient was discharged from the hospital on postoperative day 6. As of postoperative day 71, per the patient's daughter, the patient was alive but with significantly diminished mental status.

\section{Discussion}

In this case presentation we describe an unusual location for a common primary brain mass. This case study identifies the origin of a GBM related to the paracentral ventricular structures interpositioning itself between the body of the fornix and leaves of the septum pellucidum. To our knowledge this is the first documented case of a GBM found in this area.

A GBM within the limbic system structures, localized to the midline septum pellucidum and fornix with infiltration into the dorsal third ventricle, and without evidence for necrosis or hemorrhage is highly unique with respect to both location and radiographic presentation. These high-grade astrocytomas classically present in the cerebrum, localized to the deep white matter of the frontal, parietal, and temporal lobes.

On radiographic presentation, these aggressive tumors typically exhibit significant mass effect, vasogenic edema, necrosis, internal hemorrhagic products along with rim enhancement. In contrast, this GBM lesion exhibited signal homogeneity on all pulse sequences including the postgadolinium images.

A possible theory for the atypical radiographic appearance of this GBM lies in its proximity to the interventricular foramen. The foramina, bounded by the fornix and thalamus, most likely became impinged as the GBM grew in size, causing worsening subacute left ventricular hydrocephalus. Therefore, the lesion caused symptomatic mass effect prior to enlarging to a significant size where radiologic evidence for central necrosis or hemorrhage is typical.

In terms of the origin, it is plausible that the tumor may have formed within the septum pellucidum (SP). The SP, a component of the limbic system involved in consciousness, memory, and emotional response, serves as the relay station between the corpus callosum and fornix. It contains glial cells, neurons, and connecting veins to the choroid plexus. ${ }^{24}$ However, given that this area is bounded by anatomical constraints, this mass may have spread in a tail-like fashion into the fornix and third ventricle, which allows for easier expansion and growth.

Although many theories exist as to the origin of third ventricular lesions, the most commonly cited is the hypothesis that the neoplasm arises from the subependymal layer, separates early in its differentiation, and migrates toward the third ventricular cavity. However, given the lack of evidence for any subependymal cell layers on our final pathology 


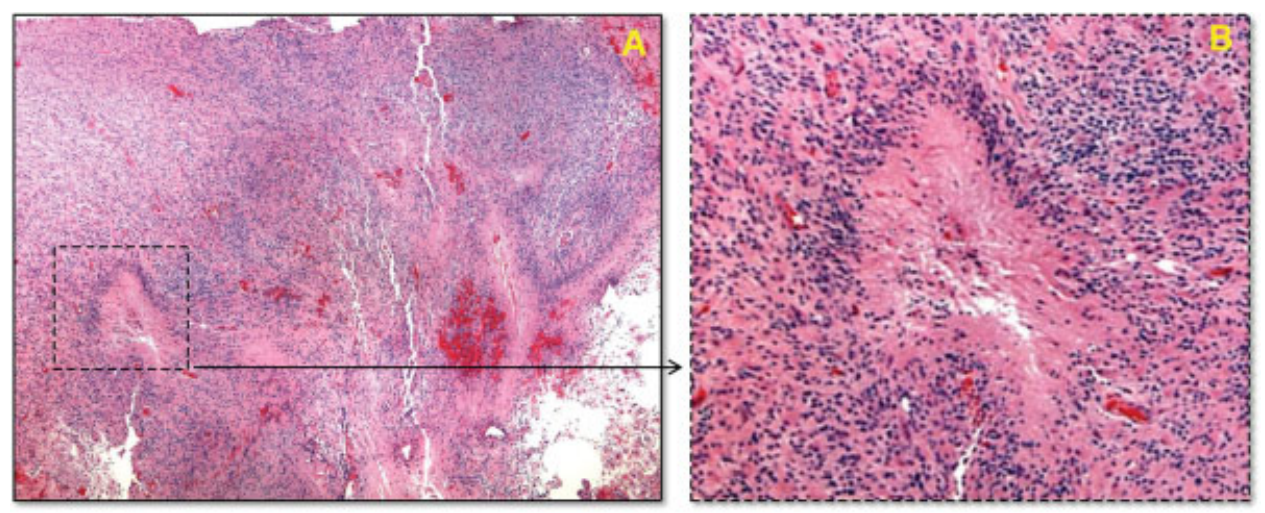

Fig. 2 Histologic examination of the excised tissue. (A) Histologic section of the resected mass (hematoxylin and eosin stain) at low magnification, showing the serpentine pattern of necrosis and surrounding hypercellularity. (B) Higher magnification of the inset area showing a focus of necrosis, with the characteristic perinecrotic pseudo-palisading pattern that occurs with glioblastoma. Red discoloration occurs due to bleeding from necroses and microvascular proliferation.

studies, it is less likely that this neoplastic lesion originated within the subependymal cell layer.

A less commonly cited hypothesis states that there may have been a primary GBM elsewhere in the brain, and the tumor cells subsequently seeded to the third ventricle via the cerebrospinal fluid. ${ }^{20}$ This possibility is also unlikely in this case, given the lack of radiographic evidence for any other neoplasms in the brain or cervical, thoracic, and lumbar spine.

Interestingly, Galli et al reported that progenitor neural cells can also be a potential source of GBMs. ${ }^{22}$ Neural stem cells from the subventricular zone migrate during injury to produce oligodendrocytes in the fimbriae fornix. ${ }^{25}$ In this fashion, the mass may have originated in the progenitor neural cells of the fornix and spread into both the septum pellucidum and the third ventricle, which could be explained clinically by the patient's impaired memory. ${ }^{11}$

\section{Conclusion}

In light of the current understanding on the origin of GBMs, we postulate that the tumor in this case may have originated in any of the structures that surround the third ventricle cavity. The origination of this mass could have been from the hypothalamus or septal nuclei. In animal model studies, septal nuclei lesions can manifest with a wide range of signs and symptoms including impairment of learning, memory, decreased arousal, reduced response rates, as well as schizophrenia and depression in both cats and rats. ${ }^{17} \mathrm{~A}$ detailed neurobehavioral assessment was not performed on our patient, and thus we are unable to evaluate any septal nuclei involvement based on lesion studies.

However, there is a lack of any evident radiographic or gross surgical abnormality to support tumor infiltration of these structures on MRI or during surgical resection. To reach a final conclusion on this hypothesis, a positron emission tomography-computed tomography scan would be needed to provide more conclusive evidence in regard to tumor infiltration of the stated neighboring anatomical structures. Moreover, GBM is also known to have a preference to spread via white matter tracts from adjacent structures-thus diffuse tensor imaging could provide more evidence as to the origin of this mass.

Given the location of the mass at the fornix, near the left foramen of Monroe, the radiographic differential diagnosis for this case included central neurocytoma, subependymal giant cell astrocytoma (commonly associated with tuberous sclerosis), or an intraventricular meningioma. The lack of marked contrast enhancement makes the latter two etiologies less likely as well. Furthermore, it is important to note that the reported natural history of septum pellucidum tumors has a predilection for the younger patient population, with a peak incidence of 10 to 12 years old. ${ }^{1}$ It is therefore important to keep a broad differential in mind because this case demonstrates that it is entirely possible for a GBM lesion to present atypically, both in terms of anatomical location as well as radiographic findings. This has very important ramifications for the clinical course as well as the management of such patients.

\section{References}

1 Mohin G, Madajewicz S, Mazione J, et al. Glioblastoma multiforme: advances in postsurgical management. Community Oncol 2006; 3(10):678-683

2 Moiyadi A, Sridhar E, Jalali R. Intraventricular gliosarcoma: unusual location of an uncommon tumor. J Neurooncol 2010;96(2): 291-294

3 Prakash O, Lukiw WJ, Peruzzi F, Reiss K, Musto AE. Gliomas and seizures. Med Hypotheses 2012;79(5):622-626

4 Rees JH, Smirniotopoulos JG, Jones RV, Wong K. Glioblastoma multiforme: radiologic-pathologic correlation. Radiographics 1996;16(6):1413-1438; quiz 1462-1463

5 Stark AM, Nabavi A, Mehdorn HM, Blömer U. Glioblastoma multiforme-report of 267 cases treated at a single institution. Surg Neurol 2005;63(2):162-169; discussion 169

6 Vougiouklakis T, Mitselou A, Agnantis NJ. Sudden death due to primary intracranial neoplasms. A forensic autopsy study. Anticancer Res 2006;26(3B):2463-2466

7 Wen PY, Kesari S. Malignant gliomas in adults. N Engl J Med 2008; 359(5):492-507 
8 Whitley RJ, Markert JM. Viral therapy of glioblastoma multiforme. Proc Natl Acad Sci U S A 2013;110(29):11672-11673

9 Lee TT, Manzano GR. Third ventricular glioblastoma multiforme: case report. Neurosurg Rev 1997;20(4):291-294

10 Glastonbury CM, Osborne AG, Salzman KL. Masses and malformations of the third ventricle: normal anatomic relationships and differential diagnoses. Radiographics 2011;31(7): 1889-1905

11 McKean-Cowdin R, Razavi P, Barrington-Trimis J, et al. Trends in childhood brain tumor incidence: 1973-2009. J Neurooncol 2013; 115(2):153-160

12 Patel DM, Schmidt RF, Liu JK. Update on the diagnosis, pathogenesis, and treatment strategies for central neurocytoma. J Clin Neurosci 2013;20(9):1193-1199

13 Kim YJ, Lee SK, Cho MK, Kim YJ. Intraventricular glioblastoma multiforme with previous history of intracerebral hemorrhage : a case report. J Korean Neurosurg Soc 2008;44(6):405-408

14 Park DeWitt J, Kim YH, Han JH, et al. Primary intracranial germ cell tumor originating from septum pellucidum that mimics central neurocytoma. J Clin Oncol 2012;30(27):e274-e277

15 Hasso A, Shakudo M, Chadrycki E. In: Lufkin RB, Bradley WG, Brant-Zawadki M, eds. MRI of the Brain III: Neoplastic Disease (The Raven MRI Teaching File series). New York, NY: Raven Press; 1991

16 Yasargil MG. Intraventricular tumors. Microneurosurgery of CNS Tumors. Vol. 4B;New York, NY: Thieme Medical Publishers; 1996: 313-342
17 Albert Lasierra P. Tumores y otros procesos de la parte anterior del III ventriculo. Neurocirugia 1999;10:27-41

18 Lejeune J-P, Le Gars D, Haddad E. Tumors of the third ventricle: review of 262 cases. [in French]. Neurochirurgie 2000;46(3):211-238

19 Villani RM, Tomei G. Transcallosal approach to tumours of the third ventricle. In: Sweet SA, ed. Operative Neurosurgical Techniques, Indications, Methods, and Results. Vol. 1, 4th ed. Philadelphia, PA: WB Saunders; 2000:862-875

20 Prieto R, Pascual JM, Roda JM. Third ventricle glioblastoma. Case report and review of literature. Clin Neurol Neurosurg 2006; 108(2):199-204

21 Ahmadloo N, Kani AA, Mohammadianpanah M, et al. Treatment outcome and prognostic factors of adult glioblastoma multiforme. J Egypt Natl Canc Inst 2013;25(1):21-30

22 Galli R, Binda E, Orfanelli U, et al. Isolation and characterization of tumorigenic, stem-like neural precursors from human glioblastoma. Cancer Res 2004;64(19):7011-7021

23 Matsuyama N, Uwano T, Hori E, Ono T, Nishijo H. Reward contingency modulates neuronal activity in rat septal nuclei during elemental and configural association tasks. Front Behav Neurosci 2011;5:26

24 Sarnat HB, Netsky MG. Evolution of the Nervous System. New York, NY: Oxford University Press; 1974

25 Steffenhagen C, Dechant FX, Oberbauer E, et al. Mesenchymal stem cells prime proliferating adult neural progenitors toward an oligodendrocyte fate. Stem Cells Dev 2012;21(11): 1838-1851 